\begin{tabular}{|c|c|}
\hline \multirow{3}{*}{ 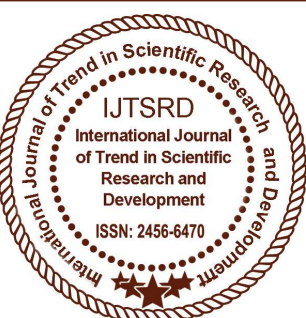 } & $\begin{array}{l}\text { International Journal of Trend in Scientific } \\
\text { Research and Development (IJTSRD) }\end{array}$ \\
\hline & Access Journal \\
\hline & ISSN No: 2456 - 6470 | www.ijtsrd.com | Volume - 2 | Issue -3 \\
\hline
\end{tabular}

\title{
A Review on Various Methods of Transformer Protection
}

\begin{abstract}
Anand Sharma
Assistant Professor, Department of Electrical Engineering, Poornima College of Engineering, Sitapura, Jaipur, Rajasthan, India
\end{abstract}

\author{
Dr. Deepika Chauhan \\ Associate Professor, Department \\ of Electrical Engineering, \\ Poornima College of Engineering, \\ Sitapura, Jaipur, Rajasthan, India
}

\author{
Vijay Kumar Mahawar \\ Final Year Student, Department of \\ Electrical Engineering, Poornima \\ College of Engineering, Sitapura, \\ Jaipur, Rajasthan, India
}

\section{ABSTRACT}

The paper present review on various transformer protection technologies and monitoring \& controlling of transformer. The paper describes the technologies by which we can protect the transformer. This includes transformer protection using PLC system, GSM module, relay, fuzzy logic, arduino etc. This paper also includes the monitoring \& controlling of transformer with the help of PLC system and single board computer. It also shows the effect of inrush current on transformer protection. Transformers are the most important equipments for electrical energy transfer in power system. Stability and security of transformer protection are important to system operation. Hence, this paper includes various technologies for the transformer protection.

Keywords: Transformer, Fault, Protection, Inrush Current, Relay, PLC, Monitoring \& Controlling

\section{INTRODUCTION}

Transformer is the vital equipment in power system network. Because of, the large number of transformers and various components over a wide area in power system, the data acquisition, condition monitoring, automatic controlling, and protection are the important issue. Transformer is a static device which transfers energy from one circuit to another without any change in the frequency Proper protection is needed for economical and safe operation of electrical power system. Transformer facing problem of inrush current, thermal overload etc. So, in this review paper all technologies and protection scheme of transformer are discussed.

Transformer is a very expensive and vital equipment which needs proper protection from fault and overload condition. Because of its relatively simple in construction, it is highly reliable equipment. This reliability, however, requires proper design methodologies, annual overhauling and different protection schemes for different possible issues. The primary goal of transformer protection is to detect faults inside the protective zone not only with sensitivity, but also with a high degree of immunity. The type of protection for the transformers varies depending on the application and the importance of the transformer. Transformers are protected primarily against fault and overload condition. Better protection schemes can provides stability, reliability and security to the power system. [2].

The main aim of this paper is to presents different technologies of transformer protection.

\section{METHODS OF TRANSFORMER PROTECTION}

\section{A. Thermal Overload Protection}

The overheating in transformer is basically due to the overloads and short circuits. In thermal over load protection system if the temperature of the transformer is rise beyond the safe value, the transformer disconnected from the system. It is done 
by the insertion of the control circuit in the secondary winding. This inserted circuit senses the temperature of the transformer and disconnected the system from the secondary winding. This control circuit again senses the temperature of the transformer and if the temperature is normal then these circuit will connect the transformer to the system. In this type of overload protection the working of control circuit is automatic. Action takes place in control circuit is depend on the temperature of the transformer [1].

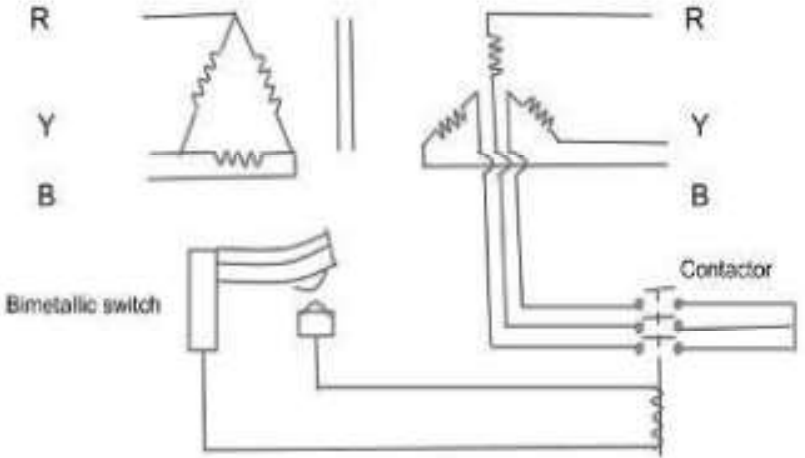

Fig.1- When transformer is overloaded [1]
As shown in figure 1, the protection system is connected to the secondary winding of the transformer. The primary winding of the transformer is delta connected and the secondary winding is star connected. The protection system is connected to the neutral point of the secondary winding. Secondary winding is connected to the three phase contactor and this contactor is connected to the bimetallic switch. In these type of switch the bimetallic strip is to convert a temperature change into

Mechanical displacement. The temperature is sense by this bimetallic switch. When the temperature of the transformer is increased the bimetallic switch sense the temperature and change it's position. The switch is connected to the contactor. Then the switch opens the contactor's contact. When the contacts are open the secondary winding of the transformer is open and the system is separate from the transformer [1].

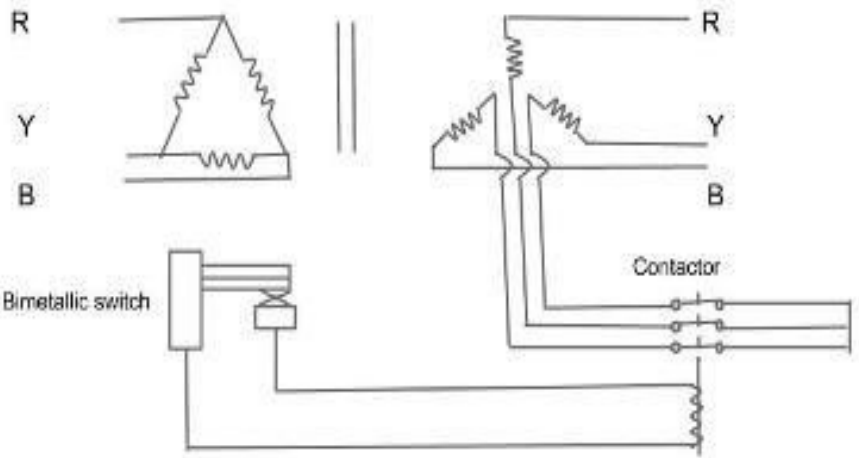

Fig. 2- When the transformer is not overloaded [1]

Under normal operating condition the contactor is closed and transformer is connected to the system. This is an automatic type protection for overload condition.

\section{A. Transformer Protection Using Arduino}

Arduino is an open-source electronics platform based on easy-to-use hardware and software. Arduino boards are able to read inputs - light on a sensor, a finger on a button, or a Twitter message - and turn it into an output - activating a motor, turning on an LED, publishing something online.

Arduino consists of both a physical programmable circuit board (often referred to as a microcontroller) and a piece of software, or IDE (Integrated Development Environment) that runs on your computer, used to write and upload computer code to the physical board.

By programming in arduino we can protect the transformer from fault. Programming is quite efficient than differential relay mechanism, so it is better to use Arduino instead of differential relay. The working of transformer is verified by Arduino every time. It senses the condition of transformer each and every second. If it founds any error then it sends commands to the circuit breakers to trip the main potential transformer. So it is the efficient and best method to protect the transformers under abnormal conditions [2]. 


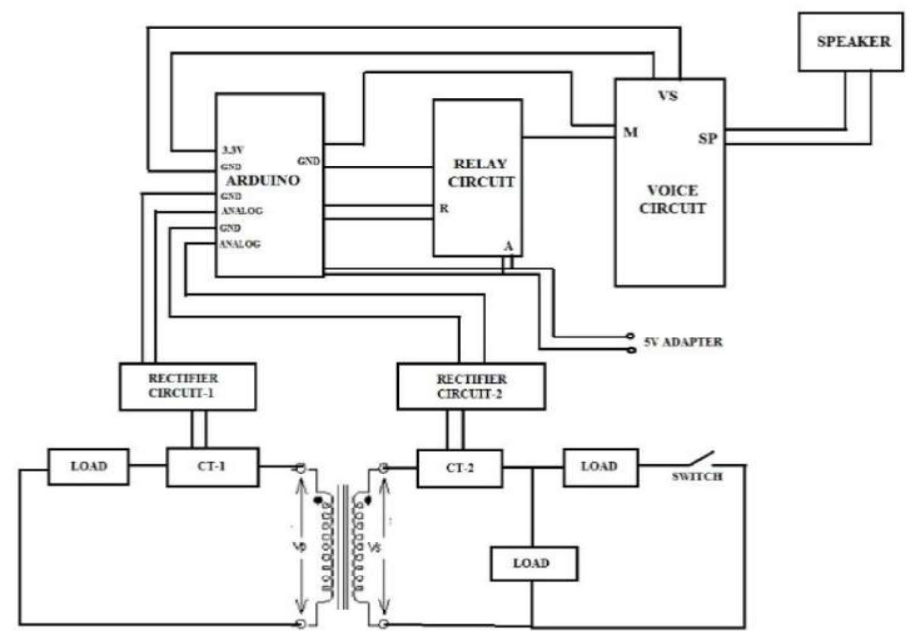

Fig 3- : Circuit diagram of differential protection of transformer using arduino [2]

Above figure shows the circuit diagram of the differential protection of transformer using arduino with voice signal. Arduino is the main component in this circuit. Arduino compares the currents in both primary and secondary winding. Two rectifiers are used in this circuit to convert the $\mathrm{AC}$ voltage into $\mathrm{DC}$ voltage for the arduino. Two current transformers also provided on both primary and secondary side to tap the currents on both sides and to give balanced voltage to the rectifier circuits. One relay circuit is connected to the arduino. Relay circuit is used to give the signal to the voice circuit, and then this voice circuit generates a voice signal.

Under normal operating condition currents on both primary and secondary side are same. So, the balanced voltages generated by the current transformers on primary and secondary side are same. These two voltages will give to the arduino. The balanced voltages generated by current transformers are rectified by the rectifiers in the circuit. Under normal operating condition these two voltages will be same in magnitude and difference is zero. So, the arduino gives no signal to the relay [2].

When fault occurs in the transformer the currents seen by the CT on primary and secondary side differs by same amount. As a result voltage sensed by the arduino from primary and secondary side differs. As there is a difference in the voltage sensed by the arduino i.e., difference is not zero. Arduino give a signal to the relay. When the relay is activated by the arduino the relay will activates the voice circuit. The voice circuit will give output predefined voice as an alert to the operator. After three consecutive voice alerts Arduino will give trip signal to the relay board and which is connected in series with the Supply will open its contacts thus the supply to the hardware setup will be disconnected.

\section{B. Transformer Protection Using PLC}

A programmable logic controller (PLC), or programmable controller is an industrial digital computer which has been ruggedized and adapted for the control of manufacturing processes, such as assembly lines, automation, or robotic devices, or any activity that requires high reliability control and ease of programming and process fault diagnosis.

These controllers are specially designed to survive in harsh situations and shielded from heat, cold, dust, and moisture etc. PLC consists of a microprocessor which is programmed using the computer language. The program is written on a computer and is downloaded to the PLC via cable. These loaded programs are stored in non - volatile memory of the PLC.

Due to wide range of PLC automation, the various types of fault in power transformer can be detected and diagnosed by using PLC system [3].

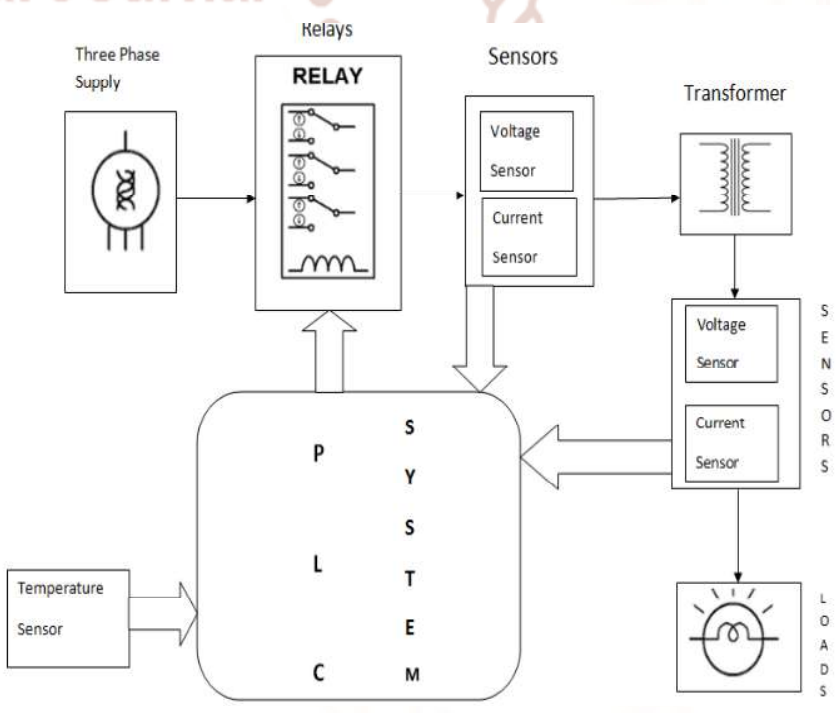

Fig.4- PLC based transformer fault detection [3]

Above figure shows the fault detection and protection of transformer using PLC system. In this system three sensors are used. First sensor is used for relay circuit, second for transformer and third is used for temperature sensing.

All the sensors sense the fault and give a signal to the PLC system. This PLC system sends a signal to the relay. 
When the PLC recognizes any increase or decrease in the level of voltage, current or temperature values the unit has been made shutdown in order to prevent it from further damage with the help of relay [3].

\section{Transformer Protection Using Differential Relay}

A differential relay is defined as the relay that operates when the phase difference of two or more identical electrical quantities exceeds a predetermined amount. The differential relay works on the principle of comparison between the phase angle and magnitude of two or more similar electrical quantities. Comparing two electrical quantities in a circuit using differential relays is simple in application and positive in action.

One of the most effective methods of protection to protect transformers is the Differential protection method by using differential relay circuits. This scheme is based on the principle of that the power input to the transformer under normal conditions is equal to the power out. By proper connection of the secondaries of current transformers (CT), under normal conditions, no current will flow into the relay coil. Whenever a fault occurs the current balance will no longer exist and relay contacts will close and release a trip signal to Circuit Breakers (CB) to operate in order to disconnect the faulty equipment [4].

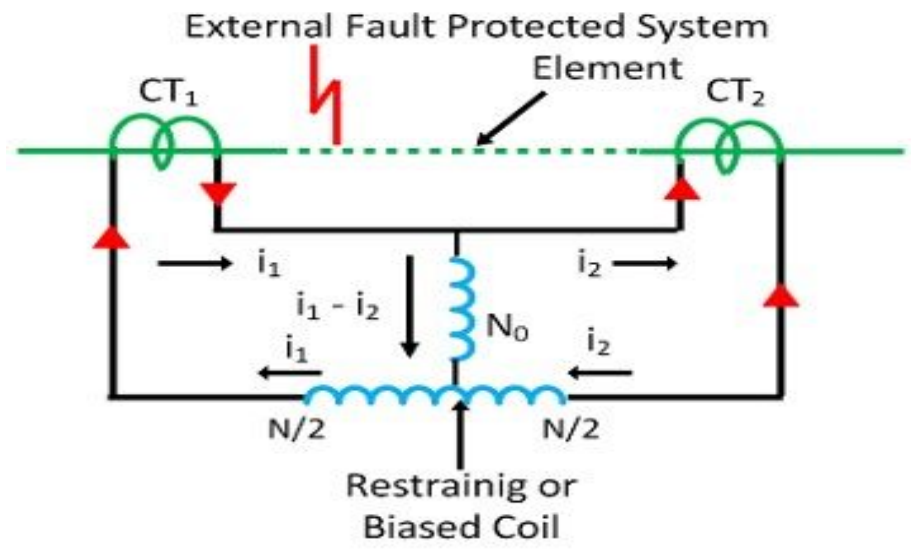

Biased or Percentage Differential Relay

circuit Globe

Fig. 5- Differential protection of transformer [4]

Above figure shows the arrangement of overcurrent relay. The desh line shows the section which is used to be protected. The current transformer is connected at both the ends of the protection zone. The secondary of the both transformers is connected in series with the help of the pilot wire. Thereby, the current induces in the CTs flows in the same direction. The operating coil of the relay is connected on the secondary of the CTs.

In the normal operating condition, the magnitude of current in the secondary of the CTs remains same. The zero current flows through the operating coil. On the occurrence of the fault, the magnitude of the current on the secondary of CTs becomes unequal because of which the relay starts operating. After that the relay sends the signal to the circuit breaker.

\section{Monitoring \& Controlling of Transformer}

Protection of transformer is very important as well as monitoring \& controlling also important for the transformer. Many transformers are damaged due to overload or fault condition, hence by incorporating monitoring \& controlling the life of transformer can be increased.

Online monitoring system consists of embedded system, GSM modem, mobile-users and GSM networks and sensors installed at transformer site Sensors are installed on transformer side which reads and measures the physical quantity from the distribution transformer and then it converts it into the analog signal. The embedded module is located at the transformer site. It is utilized to acquire process, display, transmit and receive the parameters to/ from the GSM modem. The second is the GSM module. It is the link between the embedded system and the public GSM network. The third is utility module that has a PC-based -server located at the utility control center. The server is attached to GSM modem and received transmits SMS from/to the transformer site via the GSM module [5].

\section{Conclusion and Future work}

In this paper different type of methods of transformer protection are discussed and monitoring \& controlling of transformer is also discussed. In this paper four methods of transformer protection are discussed like transformer protection using arduino, PLC system, differential relay and thermal overload protection using bimetallic switch. Firstly thermal overload protection describes the protection scheme $\mathrm{o}$ transformer from the overheating of transformer. The benefit of this method is, it is automatic protection method no manual work is required. Second is transformer protection using arduino. This protection scheme works efficiently. Sensitivity and reliability of this method is very high for faulty condition. In future, this work can also be extend using PLC. Third 
is transformer protection using PLC. PLC is used to monitor and control the voltage, current and temperature of the transformer.

PLC system makes the transformer more robust against other issues. This method makes the transformer more reliable, secure and efficient. Fourth is transformer protection using differential relay. This is the most effective method to protect the transformer from fault. This relay has a unique advantage of reducing the time of operation to a minimum for faults very close to the source where the fault current is the greatest.

More research in fault analysis and relay will result in better performance in transformer protection. If circuit counter and GSM circuit is connected then we can monitor the transformer from remote location.

\section{References}

1) Chetan S. Patil, Prashant A. Gite, Pooja B. Pawar, "Thermal overload protection of distribution transformer",Ucoer, Pune, 2014 IJEDR PP 55-58

2) Sarfaraz Nawaz Syed, S. Radhika, M.N. Sandhya Rani, "Differential current protection tof transformer using arduino with voice alert", GRIET, Hyderabad, Andhra Pradesh, 2015 International Journal of Innovations in Engineering and Technology

3) Satya Kumar Behera, Ravi Masand, Dr. S.P. Shukla, "A review of Transformer Protection by Using PLC system", Bhilai Institute : of Technology, Durg, India, 2014 IJDACR
4) Ihedioha Ahmed C., "Differential protection for power transformer using relay", Enugu state university of science and technology, enugu, Nigeria, 2016 IJTRD

5) Abdurrahman Shu'Aibu Hassan, "Monitoring and Controlling of Distribution Transformer Using GSM Module (AVR Microcontroller Based)", Sharda University, G.Noida, India, 2017 International Journal of Advanced Research in Science and Engineering

6) Piyali Sarkar, Sandhya Upreti, Sumeet Singh, "Study on methods of transformer protection", Dronacharya college of engineering, Haryana, India, 2014 IJIRT

7) Dr. Abbas H. Abbas, "Power Transformer protection by using Fuzzy Logic", University of Basrah, Iraq, 2009 IEEE

8) Nikhil P. Shetty, Yash Sachdeva, Trapti Tagare, "Transformer Monitoring System Using GSM Module", DSCE, Karnataka, India, 2016 IJIREEICE

9) Adil Naseem, Naveed Alam, "Protection of distribution transformer using arduino platform", University of Lahore, Lahore, Pakistan, 2015 IRJET

10) Pravin S Gulhane, Prof. Ch. Malla Reddy, Ajit S. Gaikwad, "Recent trends in Digital Differential protection of power transformer", Fabtech college of Engineering and research, Sangola, India, 2015 IJIERT 